\title{
fMRI study of bimanual coordination
}

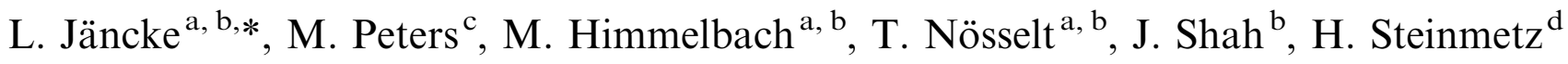 \\ ${ }^{a}$ Research Center Jülich, Institute of Medicine, Jülich, Germany \\ ${ }^{\mathrm{b}}$ Otto v. Guericke Universität Magdeburg, Postfach 4120, 39016 Magdeburg, Germany \\ ${ }^{\mathrm{c}}$ Department of Psychology, University of Guelph, Guelph, Canada \\ ${ }^{\mathrm{d}}$ Johann Wolfgang Goethe-Universität Frankfurt, Clinic for Neurology, Frankfurt, Germany
}

Received 19 August 1998; received in revised form 23 March 1999; accepted 31 March 1999

\begin{abstract}
Eleven right-handed subjects performed uni- and bimanual tapping tasks. Hemodynamic responses as measured with functional magnetic resonance imaging (fMRI) in the primary somato-motor cortex (SMC) showed that during bimanual activity the SMC contralateral to the hand taking the faster rate was more strongly activated than the SMC contralateral to hand taking the slower rate. There were no asymmetries; left SMC activation during the right fast/left slow tapping condition was comparable to the right SMC activation during the left fast/right slow condition. A given SMC showed similar activation levels for bimanual and unimanual activity (i.e. left SMC activation for right fast/left slow was similar to left SMC activation for the right fast unimanual condition). In contrast, a given supplementary motor area (SMA) showed significantly more activation for the bimanual than for the unimanual activity. In addition, an asymmetry was observed during bimanual activities: during the right fast/left slow activity, the left SMA showed more activation than the right SMA, but during the left fast/right slow activity, the right SMA was not significantly more activated than the left SMA. For unimanual activities, a clear rate effect (greater activation for faster rate) was seen in the SMC but not in the SMA. (C) 1999 Elsevier Science Ltd. All rights reserved.
\end{abstract}

Keywords: fMRI bimanual; Motor cortex; Supplementary motor area; Tapping; Handedness

\section{Introduction}

In humans, the consistent population preference given to the right hand represents a remarkable species characteristic which finds only weak antecedents in subhuman primates [28]. However, in the normal skilled use of the hands the right hand preference tends to be part of a role specialization of the two hands where consistent tasks are assigned to the right and left hands. Typically, the two hands collaborate in such a way that each hand describes different movement trajectories which fulfil complementary roles. For example, in peeling a carrot, the left hand holds and positions the carrot while the right hand performs the

\footnotetext{
* Corresponding author. Tel.: +49-391-67-14842; fax: +49-391-6714815.

E-mail address: lutz.jaencke@gse-w.uni-magdeburg.de (L. Jäncke).
}

peeling movements. The question of how the activities of the two hands are integrated can be approached at two levels. First, there is the question of how the component activities contribute to a common higher order goal. Second, there is the question of how the temporal trajectories of the two hands can be coordinated. At present, the first question is beyond the grasp of available quantitative methods. However, the second question is approachable as long as the movement onand offsets are clearly defined. Preliminary work suggests that the timing demands of the two hands are met as a function of handedness. In general, the timing of the movements of the right hand dominates and the timing of the movements of the left hand is intercalated. However, if the task demands require the nondominant hand to perform the more complex and or faster movements, then the timing demands of that hand predominate [37]. Thus, the mechanisms by which handedness and task demands interact deter- 
mine which hemisphere takes the leading role in timing the chain of movements into which the component activities are integrated.

The work directed at understanding the brain structures involved in bimanual integration is in its infancy. However, the supplementary motor cortex (SMA) has been implicated in bimanual integration in animals $[7,54]$ and humans $[8,20,29]$. One goal of our study was to see whether a simple bimanual tapping task can be adapted to a fMRI analysis, so that putative asymmetrical activations can be observed in the primary and supplementary motor cortices in humans. It was hoped that such an approach could contribute a preliminary understanding of the mechanisms involved in the timing of bimanual and temporally integrated responses. The bimanual task chosen was very simple: subjects had to tap twice with one hand for each single tap in the other. Integration was provided by the requirement that the second tap in the faster hand coincided with the single tap in the slower hand. What this task lacks in complexity is compensated for by its easily quantifiable nature, it is precise timing demands and the fact that the activities of the two hands add to yield a rhythmical structure which emerges through the separate contributions of the two hands. The task thus has-albeit in simplified form-the essential elements of normally occurring bimanual tasks $[34,35]$. In addition, evidence shows that the task is not performed equally well when the two hands exchange roles: performance in right-handers is better when the right hand taps twice for each single tap in the left hand as opposed to the converse condition [36]. Thus, task performance shows an asymmetry. Finally, the task has some relevance to more complex aspects of behavior and thus has 'ecological validity'. For instance stutterers show different performance patterns on this task than non-stuttering righthanders [51,53] and children with learning disabilities tend to perform the bimanual task significantly and disproportionately worse than single handed tapping when compared to control children who were not learning disabled [4,55]. Finally, skilled musicians perform more accurately on the task than control subjects, by showing less variability in intertap intervals. However, the role of quality of performance is not of interest in the present study; one could even argue that highly skilled subjects would be less suitable for the fMRI study if their greater ease in performing the task should express itself in a smaller fMRI signature.

\section{Method}

\subsection{Subjects}

Eleven healthy right-handed adults were tested (8 men and 3 women, ages 22-37]. All were screened for neurological impairments, and were found to be healthy. The subjects were right-handed, as determined by the criterion of consistent right hand preferences for all items of the Annett hand preference questionnaire [2].

\subsection{Procedure}

There were six experimental conditions:

1. LFRS: where the left hand tapped twice for each tap with the right hand (left fast/right slow).

2. RFLS: where the right hand tapped twice for each tap with the left hand (right fast/left slow).

3. LF: where the left hand tapped at the same rate as LF in the LFRS condition, but alone.

4. RF: where the right hand tapped at the same rate as RF in the RFLS condition, but alone.

5. LS: where the left hand tapped at the same rate as LS in the RFLS condition, but alone.

6. RS: where the right hand tapped at the same rate as $\mathrm{RS}$ in the LFRS condition, but alone.

The first two conditions are the bimanual conditions. The four unimanual conditions allow comparison of the cortical activation for a given hand and tapping rate for bimanual vs unimanual activity. For example, one could compare the left cortical activation for the RF in the RFLS condition with activation when only the right hand was active by tapping the RF rate.

Because the fMRI conditions do not allow metal components in the tapping apparatus, subjects' responses where registered by key presses which interrupted an optic fibre light beam. This allowed registration of 'on' and 'off' responses. The wrist as well as all of the inactive fingers (tapping was only done with the index finger) were taped so that they could not participate in the tapping movement. All subjects were first given the opportunity to practice and the practice was used to instruct the subjects in the task and to ascertain that they could perform the task as required. The six conditions were performed in sequence, as listed. Subjects were instructed to keep their eyes open throughout the scanning series. During the scanning session room lights were dimmed. In each experimental condition a series of 85 images was acquired. Each series consisted of multiple periods of 'baseline' (OFF, rest), during which subjects heard only the ambient machine noise but did not perform the motor task, alternating with periods of 'activation' (ON, task). During the rest conditions subjects received a constant light via goggles fitted into the head coil. Immediately after switching off of this light the subjects were to start the required movement conditions while switching on of the light indicated the start of the next rest condition, during which no move- 
ment should be made. Tapping rate was monitored during the scanning session applying a fiber optic system attached to a laboratory computer counting the taps. Each scanning series began with 5 baseline images ( $15 \mathrm{~s}$ interval) allowing signal equilibrium to be reached and an initial baseline to be established, followed by 80 images during which 'activation' alternated with 'baseline' every $15 \mathrm{~s}$ ( $30 \mathrm{~s} /$ cycle, 10 images/ cycle, 8 cycles). The total duration of each image series was about $6 \mathrm{~min}$. The tapping was self-paced.

\subsection{Scanning procedures}

Functional MR images were acquired using a 1.5 Tesla Siemens MRI system (SIEMENS Magnetom Vision, Erlangen, Germany), equipped with echo planar imaging (EPI) capabilities and a standard radiofrequency (RF) head coil was used for transmission and reception. Sequences with the following parameters were applied: echo planar imaging EPI, TR $=3 \mathrm{~s}$, $\mathrm{TE}=66 \mathrm{~ms}, \quad \mathrm{FOV}=200 \times 200 \mathrm{~mm}, \alpha=90^{\circ}$, matrix size $=64 \times 64, \quad$ in plane resolution $=3.125 \times 3.125 \times 3.0 \mathrm{~mm}^{3}$. Using a midsagittal scout image, 16 axial slice positions $(0.1 \mathrm{~mm}$ interslice gap) were oriented in the anterior-posterior commissure (AC-PC) plane and the uppermost slice was aligned $2 \mathrm{~mm}$ below the vertex, thus covering the motor cortex and adjacent motor areas (premotor cortex and supplementary motor cortex). In addition, 3D images of the entire brain were obtained by using a strongly T1-weighted gradient echo pulse sequence (fast low-angle shot) with the following parameters: $\mathrm{TR}=40 \mathrm{~ms}, \mathrm{TE}=5 \mathrm{~ms}, 40^{\circ}$ flip angle, 1 excitation per phase encoding step, $F O V=25 \mathrm{~cm}$, matrix size $=256 \times 256,128$ sagittal slices with $1.25-\mathrm{mm}$ single slice thickness.

\subsection{Image analysis}

Image analysis was performed on ULTRA workstations (Sun Microsystems) using MATLAB (Mathworks Inc., Natiek, MA, USA) and SPM96 [1216]. First, the 80 volume images of each condition were automatically realigned to the first image to correct for head movement between scans. The images of the six conditions were then coregistered and transformed into a standard stereotactic space corresponding to the atlas of Talairach and Tournoux [47], using the intercommissural line as the reference plane for the transformation. The spatial normalization involves linear and non-linear transformations to match each scan to a reference image that already conforms to the standard brain. Within this normalization, voxels were slightly smoothed to achieve isotropic voxels representing $4 \times 4 \mathrm{~mm}$ in the $x$ and $y$ dimensions, with an interplanar distance of $4 \mathrm{~mm}$. Voxels that had values greater than 0.8 of the volume mean in all the images were selected to restrict that analysis to intracranial regions. The effects of global (whole volume) activity and time were removed as confounds, using linear regression and sine/cosine functions (up to a maximum of 2.5 cycles per 80 scans). Removing the latter confounds corresponds to high-pass filtering of the time series to remove low frequency artifacts which can arise due to aliased cardiorespiratory and other cyclical components. Images were then smoothed with an isotropic Gaussian kernel ( $8 \mathrm{~mm}$ full-width half-maximum) in order to meet the statistical requirements for using the 'General Linear Model' and to account for interindividual differences in sulcal and gyral landmarks.

\subsection{Statistical parametric mapping}

Significantly activated voxels were searched for by using the 'General Linear Model' approach for timeseries data suggested by Friston and colleagues $[12,15,57]$. For this we defined a design matrix comprising contrasts modeling the alternating periods of 'baseline' and 'activation' using a delayed box-car reference vector accounting for the delayed cerebral blood flow after stimulus presentation. The resulting statistical parametric map of $\mathrm{t}$ statistic generated for each voxel was transformed to a map of corresponding $Z$ values, thresholded at a $Z$ value of $3.09(P=0.001$ uncorrected for multiple comparisons) and a spatial extent criterion of $P<0.05$ (corrected for multiple comparisons which corresponds to 15 consecutively activated voxels) [40]. The activated voxels surviving this procedure were superimposed on 'SPM brain projections' and on individual stereotactically normalized MR anatomical scans. With the aid of published Talairach-coordinates, taken from Roland and Zilles [43] and prominent sulcal landmarks (precentral, cen-

Table 1

Self-paced tapping rates for the bimanual and single conditions, with standard deviations

\begin{tabular}{lll}
\hline Condition & taps/s & sd \\
\hline Left Fast (LF) $^{\mathrm{a}}$ & 2.54 & 0.41 \\
Right Fast (RF) $_{\text {RF/LS, right hand }}$ & 2.93 & 0.33 \\
LF/RS, left hand & 2.71 & 0.28 \\
RF/LS, left hand & 2.53 & 0.32 \\
LF/RS, right hand & 1.29 & 0.16 \\
Ratio right/left for RF/LS & 1.14 & 0.16 \\
Ratio left/right for LF/RS & 2.11 & 0.24 \\
\hline
\end{tabular}

\footnotetext{
${ }^{a}$ Left hand moving fast at self-paced speed, single hand moving.

${ }^{\mathrm{b}}$ Speed of the right hand when right taps twice for each single left tap, bimanual condition.

${ }^{\mathrm{c}}$ Ratio of fast tapping hand/slow tapping hand in the bimanual condition (ideal ratio is 2 ).
} 

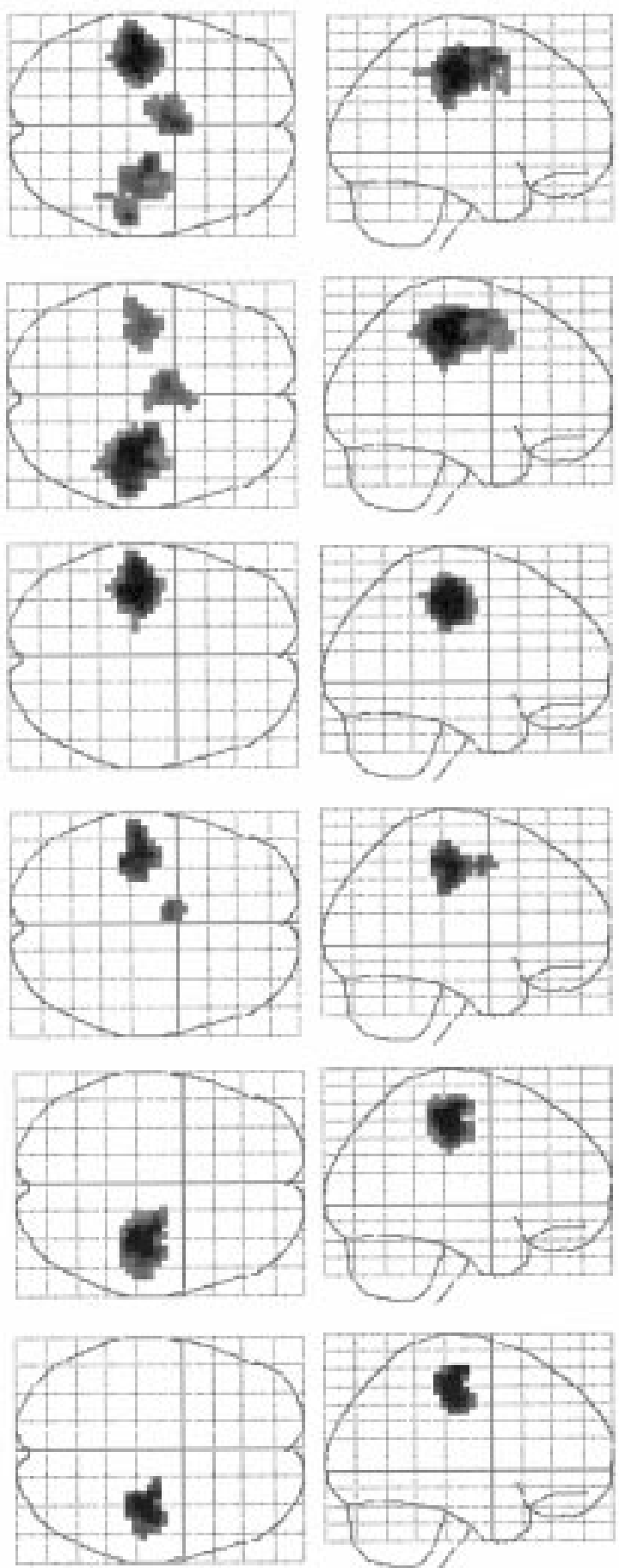

above

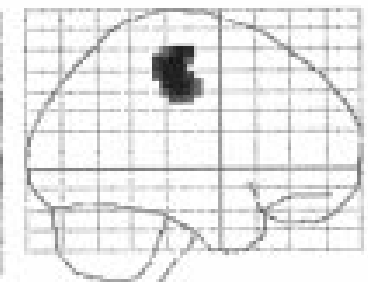

right

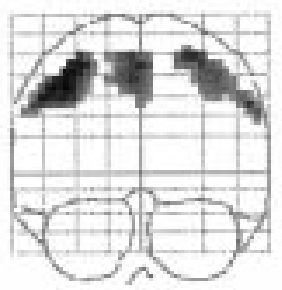

RF-LS

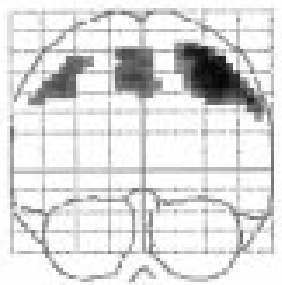

RS-LF

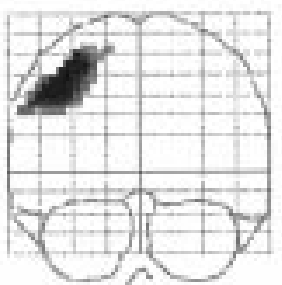

RF

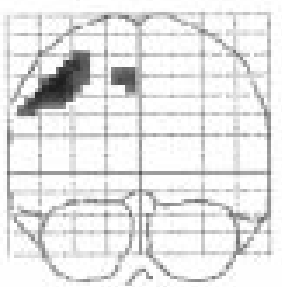

RS
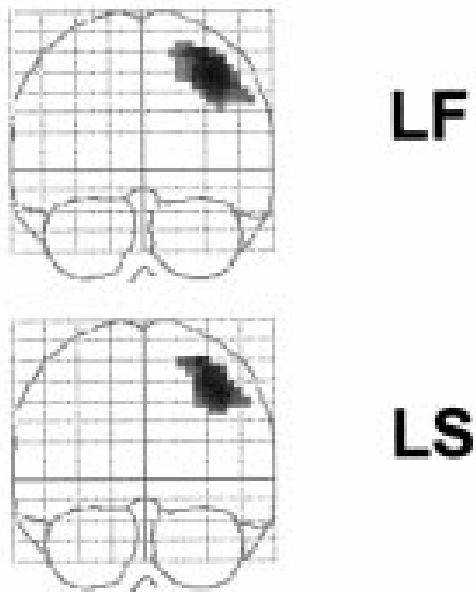

LS

Fig. 1. This illustrates the location of the activated voxels, separately for the different tasks, in a so-called 'glass brain'. There are three perspectives for each condition; a view from the right side of the brain, a view from behind (left side on the left), and a view from above (superior half represents the left half of the brain). The line where the major horizontal and vertical axes intersect is defined by the anterior commissure.

tral, and postcentral sulci [58]) clusters of activated voxels were assigned according to their center of mass activity to the following regions of interest: somatomotor cortex (SMC: comprising M1 and S1) and supplementary motor cortex (SMA). For these clusters numbers of significantly activated voxels relative to baseline were determined. In order to present the overall pattern of activation across subjects the stereotactically transformed functional data sets were pooled across the whole group (Table 1). As for the individual 


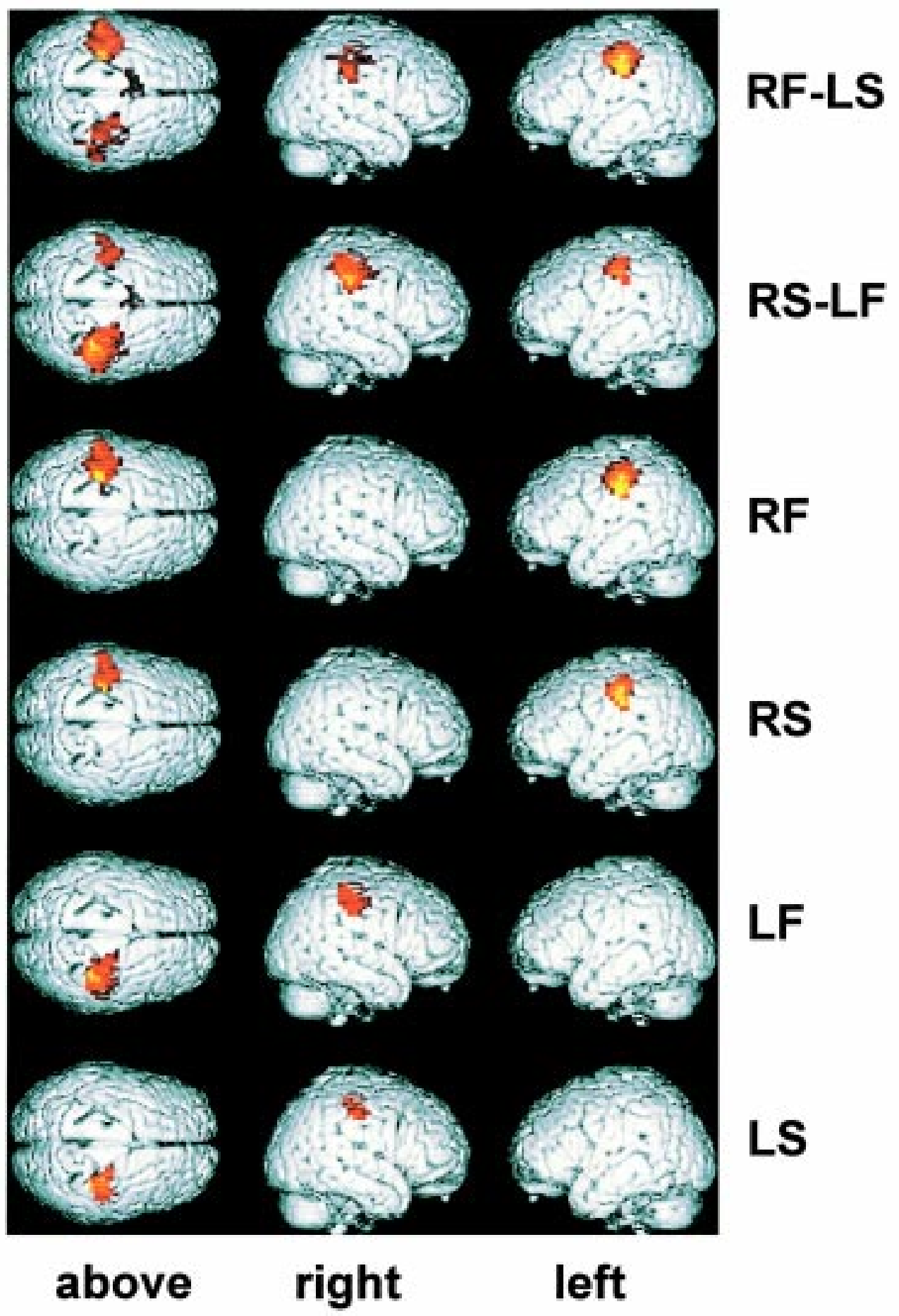

Fig. 2. Shows a view of the reconstructed brain with surface locations of the activity. Lighter color indicates higher levels of activation. Note the small area denoting the SMA, and showing activation close to the midline on the left side for both the RFLS and the LFRS condition.

analyses, only voxels passing a height threshold of $z=3.09$ and a cluster threshold of $P<0.05$ (corrected for multiple comparisons) were considered significant. The activated voxels surviving this procedure were superimposed on 'SPM-brain-projections' (Fig. 1) and a rendered $\mathrm{T} 1$ standard brain (Fig. 2).

\section{Results}

\subsection{Motor performance}

All subjects were capable of performing the tasks. The average tapping speeds can be seen in Table 2 . 
Table 2

Anatomical regions that showed significant hemodynamic responses in the group analysis

\begin{tabular}{llrrrrr}
\hline Task $^{\mathrm{a}}$ & Anatomical region & \multicolumn{1}{c}{$x$} & $y$ & $z^{\mathrm{c}}$ & $Z_{\text {-value }}^{\mathrm{d}}$ & Volume $(\mathrm{ml})^{\mathrm{e}}$ \\
\hline RF-LS & L SMC & -40 & -20 & 48 & 7.63 & 10.62 \\
& R SMC & 28 & -16 & 60 & 6.65 & 8.00 \\
\multirow{2}{*}{ RS-LF } & SMA & 0 & 0 & 56 & 6.40 & 6.40 \\
& L SMC & -40 & -16 & 48 & 6.20 & 3.97 \\
& R SMC & 32 & -28 & 52 & 6.01 & 16.06 \\
$\mathrm{RF}$ & SMA & -4 & -4 & 48 & 6.20 & 6.40 \\
RS & L SMC & -44 & -20 & 44 & 7.49 & 11.71 \\
& L SMC & -40 & -20 & 48 & 6.42 & 6.91 \\
LF & SMA & -8 & -4 & 48 & 4.65 & 0.96 \\
LS & R SMC & 40 & -24 & 48 & 7.57 & 12.74 \\
& R SMC & 40 & -24 & 48 & 5.96 & 6.72 \\
& SMA & 0 & -8 & 44 & 5.08 & 0.83
\end{tabular}

${ }^{a}$ Task: RF-LS: bimanual tapping with right hand fast and left hand slow tapping, RS-LF: bimanual tapping with right hand slow and left hand fast tapping, RF: unimanual tapping with right hand fast tapping, RS: unimanual tapping with right hand slow tapping, LF: unimanual tapping with left hand fast tapping, LS: unimanual tapping with left hand slow tapping.

${ }^{\mathrm{b}}$ Anatomical region: L, R: left and right hemisphere; SMC: somato-motorcortex; SMA: supplementary motor area.

${ }^{\mathrm{c}} x, y, z$; stereotactic coordinates in $\mathrm{mm}$ according to the anatomical atlas of Talairach and Tournoux [47].

${ }^{\mathrm{d}} Z$-value: peak activation of the given cluster.

${ }^{\mathrm{e}}$ Volume: size of activated volume.

The important aspect of these numbers is, first, that tapping rates during the single hand and bimanual conditions did not differ significantly. For example, the rate for the left hand tapping slowly during the right fast/left slow condition was comparable to the left hand slow rate in the unimanual condition. This latter point is important because it avoids a possible confounding of single vs bimanual task activation patterns with rate effects. However, the subjects tapped slightly faster in the RF/LS condition than in the LF/RS slow condition $(F=10.6, p=0.01)$. This confirms previous observations that righthanded subjects tend to perform the $\mathrm{RF} / \mathrm{LS}$ condition slightly faster than the LF/RS condition. However, in terms of the questions asked in this study, the rate difference of $0.15 \mathrm{taps} / \mathrm{s}$ is negligible.

\subsection{Group analysis of cortical activations}

An overall qualitative impression of the mean fMRI responses can be seen in Figs. 1 and 2 as well as in Table 1. In Fig. 1, each condition is shown from three different perspectives. Each presentation is constructed as if it were a 'glass brain' where each activated voxel is indicated by a region of darkness. The reader is reminded that each of the voxels is defined as significantly active relative to the overall activity in the brain. The first picture for the 'right fast/left slow' condition represents a look at the transparent brain from the right side of the brain. The area of significant activation lies in the region of the primary somato-motor cortex (SMC). In the second presentation we view the brain from above, and we can see that there is activation in the SMCs of the left (above) and the right (below) hemisphere. The single more central area of activation is in the area of the supplementary motor area (SMA), and it can be seen that there is somewhat more activity in the SMA in the left half of the brain in both of the bimanual conditions. It can further be seen that the right SMC is more broadly activated than the left SMC in the 'left fast/right slow' condition. At least in these representations, no similarly obvious pattern is seen for the 'right fast/left slow' condition. The single hand movements reveal a unilateral pattern of activation, such that only the SMC opposite of the active hand is significantly activated. There is a further, and quite clear effect: there is more activation for fast movements than for slow movements, indicated by larger areas of activation for the 'fast' condition. This is seen quite clearly in both hands. This is a 'rate' effect, which has also been seen in other studies where the unimanual performance of a hand that tapped quickly was compared to a slow tapping rate. A different perspective can be gained when the activity is mapped on a reconstructed 'standard brain', with location and intensity of the activation indicated by color (Fig. 2). The lighter the color, the more intense the activation. In this representation, there is very little visible activation in the right SMA, even during bimanual activities.

\subsection{Statistical comparison of the activated voxels during bimanual activity in SMC and SMA on the basis of the individual analyses of $f M R I$ responses}

A brief comment needs to be made about the question of how to treat data cells for which the number of activated voxels did not exceed the criterion threshold. The problem is best illustrated with an example. When the right hand taps quickly, the contralateral SMC in each of the subjects showed significant activation. However, in the SMC ipsilateral to the quickly tapping hand, only three subjects showed significant, low level, activation. In a statistical comparison between the SMC ipsi- and contralateral to the active hand, should one consider the measurement for the 8 subjects who showed no significant activation as 'missing values'? This seemed logically inconsistent because in such cases the lack of activation is meaningful in terms of known anatomical and functional information. We have chosen to treat the zero number of activated clusters in each such case as statistical datum. The average number of activated voxels, the standard error of the mean of that measure, and the number of subjects in each condition who met the minimum criterion of a 
Table 3

Mean number and standard error of the mean of activated voxels in the left and right SMC and SMA as function of the different task conditions. Note the overall smaller activations in the SMA, and the low level of activation in the SMA for the unimanual as opposed to the bimanual conditions. Bold type indicates contralateral hand for SMC in the unimanual condition, and the fast hand contralateral to the SMC in the bimanual condition; $n$ indicates the number of individuals who exceeded the threshold of 15 activated voxels for each condition and brain area

\begin{tabular}{|c|c|c|c|c|c|c|c|c|}
\hline \multirow[t]{2}{*}{ Task } & \multicolumn{4}{|l|}{$\mathrm{SMC}$} & \multicolumn{4}{|l|}{ SMA } \\
\hline & Left & $n$ & Right & $n$ & Left & $n$ & Right & $n$ \\
\hline RFLS & $204.9 / 19.0$ & 11 & $122.3 / 26.1$ & 11 & $44.0 / 8.5$ & 8 & $33.6 / 8.2$ & 8 \\
\hline RSLF & $115.0 / 15.8$ & 11 & $216.7 / 14.2$ & 11 & $37.2 / 6.5$ & 8 & $34.6 / 8.3$ & 8 \\
\hline RF & $202.1 / 9.8$ & 11 & $3.9 / 2.2$ & 1 & $10.3 / 2.9$ & 5 & $6.2 / 2.8$ & 3 \\
\hline RS & $113.7 / 28.0$ & 9 & $9.2 / 4.6$ & 2 & $21.4 / 6.0$ & 7 & $22.5 / 7.6$ & 5 \\
\hline $\mathrm{LF}$ & $38.7 / 16.7$ & 6 & $179.3 / 14.0$ & 11 & $14.2 / 5.8$ & 3 & $15.9 / 6.5$ & 3 \\
\hline LS & $1.8 / 1.8$ & 1 & $111.9 / 18.5$ & 10 & $12.8 / 5.2$ & 4 & $16.1 / 7.9$ & 4 \\
\hline
\end{tabular}

cluster of at least 15 activated voxels are shown in Table 3.

\subsection{Comparison of the activation of the brain half contra- and ipsilateral to the fast moving hand, during the bimanual task}

In order to compare the activation patterns during bimanual activity in a single analysis, all values within each of the two motor areas (SMC and SMA) were expressed relative to the overall mean activation for the four conditions. For instance, the mean activated $n$ of voxels, averaged across Left SMC RFLS, Left SMC RSLF, Right SMC RFLS, Right SMC RSLF during bimanual activity was 163.6. Each value for each condition was then expressed as percentage of this average. The same was done for the SMA, where the overall average $n$ of activated voxels was 37.4.

The resulting data set was analysed with an ANOVA for repeated measures (see ANOVA table in App. A), with repeated measures over Side of the brain (left vs right), Condition (RFLS vs RSLF), and brain area (SMA vs SMC). The following clear effects were observed (see ANOVA table, App. A): A significant interaction between Side $\times$ Condition $(p<0.0001)$ suggests that it matters whether a given side guides the slow or fast rate of movement. This has to be qualified by a triple interaction between Side $\times$ Condition $\times$ Brain Area $(p<0.004)$ which reflects the fact that activation in the left and right SMC depends on whether the SMC guides the fast or slow movement, there is relatively more activation in the left SMA when it guides the fast movement but this does not hold for the right SMA. We therefore see a functional asymmetry in the SMA that is not present in the SMC.

\subsection{Comparison of the SMA and the SMC during bimanual and unimanual fast hand movement}

The SMC and the SMA differed in another response pattern as well, as can be seen in Fig. 4. In this case, the activation for each of the contrast pairs (e.g. left SMC RFLS vs left SMC RF) was averaged and the individual averages were expressed as a percentage of this average. If the bimanual fast condition involved the same level of activation as the unilateral fast condition for the brain half contralateral to the fast hand, then both should be close to $100 \%$.

The SMC appeared insensitive as to whether the SMC guided the fast tapping rate in the unimanual or bimanual condition, and this was the case for the SMC in the left brain half as well as in the right brain half. In contrast, the SMA was more strongly activated by the fast hand in the bimanual condition than in the fast hand in the unimanual condition, and this effect was more pronounced for the left SMA than for the right SMA. This was indicated (see Appendix 1b) by a significant three way interaction of Condition $\times$ Side of Brain $\times$ Brain Area $(p<0.026)$ which qualified the single effects of Condition $(p<0.004)$ and Condition $\times$ Side $(p<0.001)$.

\subsection{Rate effects for the unimanual conditions}

There was a clear rate effect, such that the SMC contrateral to the moving hand is more active when the hand moves more quickly (see ANOVA table in Appendix 1c). The rate effect was similar for the right and left SMC areas, with a significant effect of Rate $(p<0.001)$. The analysis was only meaningful for the SMC because of the low levels of activation in general for the SMA, during unimanual movement.

\section{Discussion}

The principal objective of this study was to examine the relation between handedness and patterns of activation in SMC and SMA. The location of SMA in the current study is consistent with anatomical information $[39,43,49]$. The location of the SMA so very close to the midline makes it somewhat difficult to find a clear fMRI delineation in terms of a left/right portion in the left and right hemispheres. The statistical analysis did suggest a clear left-sided advantage for the RFLS condition, but an uncertain activation pattern with regard to the LFRS condition. Overall, the activated SMA area was relatively small compared to the surprisingly large area of activation in the SMC. It is possible that the bimanual task we used does not fully engage the SMA; at this point numerous functions have been suggested for the SMA, and it is likely that 


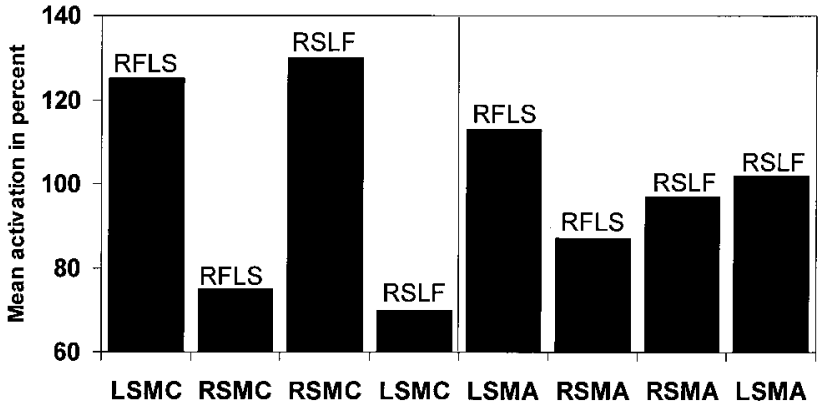

Each pair of bars shows first the cortex contralateral and then the cortex ipsilateral to the fast moving hand

Fig. 3. The average $n$ of activated voxels for each task (i.e. RFLS) for the two brain areas indicated (i.e. LSMC and RSMC) is calculated. Each individual task is then compared to that average. In the SMC, activation is clearly higher in the SMC contralateral to the fast hand, and symmetrical for the left and right SMC. In the SMA, there is no such pattern.

not all of them are equally strongly engaged in the sort of bimanual tapping task used here. We have previously referred to the element of bimanual integration as one of the task features which had been related to SMA function, both in clinical studies in humans. It is not clear whether another putative function of the SMA, that of sequencing movement, or a possible role of suppressing mirror movements $[7,8]$ is of greater importance. The role of the SMA in sequential movement has been stressed by a number of researchers $[18,19,31,33,48]$. In our task, there were two aspects of sequencing: first, the sequencing of movements within a hand and, second, the sequencing of movements between hands. In principle, the SMA should respond strongly to tasks that involve both sequencing and bimanual integration. A possible confound of sequencing lies in the necessity of providing a time signature to the sequence $[9,10,24,25]$. Finally, there is the question of whether an activity is self-paced or whether an external pacing source is provided. There is considerable agreement in the literature that the SMA is involved when sequences are voluntary/self-initiated $[6,21,26,32,41]$. The self-initiation role can also be related to the 'intentionality' aspect of motor function that is guided by the SMA, a role anticipated by Goldberg [17]. Summarizing these observations, it appears that our task did involve all of these elements, but, of course, it is not at all obvious which of the elements is of cardinal importance. In view of the relatively circumscribed activation of the SMA, our next goal will be to see how we can modify the task so that more substantial effects can be observed.

The data do make it clear that the general pattern of SMA activation is not comparable to the pattern of SMC activation. In the case of bimanual activity, both hemispheres show marked SMC activation, with clear lack of asymmetry, and an equally clear effect of rate,

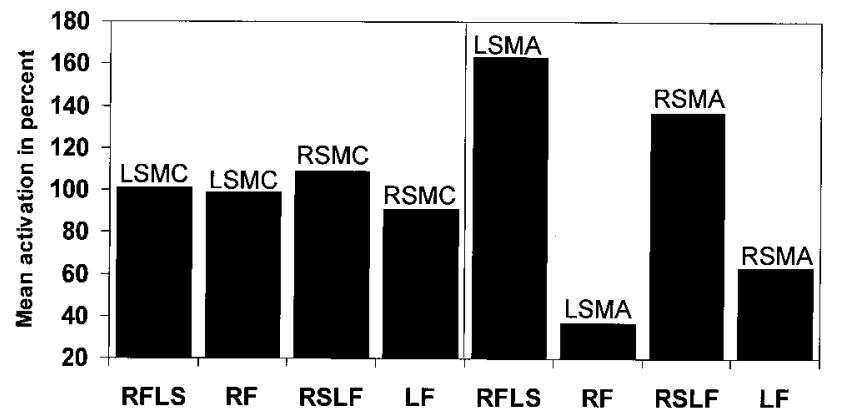

LSMC, RFLS = Bar shows activation in LSMC for RFLS $\mathrm{LSMC}, \mathrm{RF} \quad=$ Bar shows activation in LSMC for RF

Fig. 4. A similar approach as in Fig. 3, but here the average for the cortex contralateral to the fast hand in the bimanual (e.g. RFLS) and unimanual (e.g. RF) is used as reference. In the SMC, the cortex contralateral to the fast hand is activated similarly, regardless of whether the hand moves by itself or in the bimanual condition. In contrast, the SMA shows much less activation for the contralateral unimanual activity.

such that the SMC contralateral to the faster hand is more strongly activated. In the case of the SMA, there is a comparable pattern when the right hand moves quickly and the left hand slowly, with significantly greater activation in the left SMA than the right SMA, but there is no equivalent effect for the right SMA when the left hand moves quickly (Fig. 3). The SMA activations appear to favor the left hemisphere (see also Figs. 1 and 2), and this is consistent with a general model that posits a functional asymmetry in righthanders, where the left hemisphere is more prominently involved in motor planning than the right hemisphere [1,27,35].

Another difference between SMC and SMA activation is captured in Fig. 4, which shows that the SMC shows similar levels of activation to the contralateral fast hand, regardless whether that hands moves by itself, or together with the other hand during the bimanual task. In contrast, the level of activation in the SMA is clearly higher when the contralateral fast hand moves in the bimanual condition, and shows little activation when the contralateral fast hand moves by itself, in the unimanual condition. This difference confirms that the SMA is more responsive to bimanual than to unimanual activity. The fact that the SMC activation does not differentiate between uni- and bimanual faster rate tapping can be interpreted as confirming that the primary-somato-motor cortex represents the final outflow of motor responses, and is not itself involved in the integration or planning of bimanual activity.

Finally, the data also confirm a strong rate effect for the SMC, in the unimanual condition, such that activations are considerably stronger for the faster tapping rate. Because SMA activation to unimanual activity was altogether weak, no such comparison could be 
made for the SMA. The rate effect confirms previous work $[3,22,23,42,44,45,50]$. The rate effect likely signifies more than just the difference in timing and motor exertion required by the two paces; in objective terms, even the faster rate is considerably slower than the maximum tapping rate of which human subjects are capable. The literature, with some exceptions [30] agrees that the relation between internal interval and variability of internal intervals is not linear and continuous $[5,11,38,46,52,56]$. More recently, and in support of older findings in the psychophysical literature, Jäncke et al. [22] have established a nonlinear relation between fMRI activation and tapping rate. It is quite possible that the markedly different response to the rate differences in this study are as large as they are because the faster rate represents a 'semi-automatic' type of movement production while for the slower rate, each movement is produced deliberately and individually.

\section{Acknowledgements}

Please address correspondence to Dr Lutz Jäncke, Institute of General Psychology, Otto-von-GuerickeUniversität Magdeburg, Lennéstr. 6, D-39112 Magdeburg, Germany. Dr Jäncke was supported by a grant by the Deutsche Forschungsgesellschaft (DFG JA 737/7-1), and Dr Peters was supported by Natural Sciences and Engineering Research Council of Canada Grant A 7054.

\section{Appendix A}

ANOVA for comparison of the activation of SMA and SMC during bimanual activity: repeated measures over Brain Area (SMA vs SMC) $\times$ Side (Left vs Right Brain Half) $\times$ Condition (contralateral to the fast hand vs ipsilateral to the fast hand). ETA; effect size in terms of percent of variance accounted for; Power; statistical power, maximum 1.00; alpha level set at 0.05 .

\begin{tabular}{lllllll} 
& df & MS & $F$ & $p$ & ETA & Power \\
\hline Side $\times$ Condition & 1 & 24,573 & 31.5 & 0.0001 & 0.755 & 0.999 \\
Error & 1 & 791 & & & & 0.004 \\
Side $\times$ Condition $\times$ Brain Area & 1 & 11,592 & 13.8 & 0.580 & 0.917 \\
Error & 1 & 839 & & & &
\end{tabular}

\section{Appendix B}

ANOVA for comparison of SMA and SMC activation while the contralateral hand moves quickly in the bimanual and unimanual condition: Condition $($ RFLS vs RF) $\times$ Side (left vs right) $\times$ Brain Area (SMC vs SMA).

\begin{tabular}{|c|c|c|c|c|c|c|}
\hline & $\mathrm{df}$ & MS & $F$ & $p$ & ETA & Power \\
\hline Condition & 1 & $\begin{array}{r}65,762 \\
\end{array}$ & 13.7 & 0.004 & 0.578 & 0.914 \\
\hline Condition $\times$ Side & $\begin{array}{r}10 \\
1\end{array}$ & $\begin{array}{r}4802 \\
43,610\end{array}$ & 18.8 & 0.001 & 0.653 & 0.974 \\
\hline Error & 10 & 2314 & & & & \\
\hline Condition $\times$ Side $\times$ Area & 1 & 6282 & 6.6 & 0.026 & 0.400 & 0.642 \\
\hline Error & 10 & & & & & \\
\hline
\end{tabular}

\section{Appendix C}

ANOVA for comparison of fast vs slow rate movements in the SMC : Side (left vs right SMC) $\times$ Condition (hand performs at fast vs slow rate).

\begin{tabular}{lllllll} 
& df & MS & $F$ & $p$ & ETA & Power \\
\hline $\begin{array}{l}\text { Condition } \\
\text { Error }\end{array}$ & 1 & 66,690 & 21.6 & 0.001 & 0.683 & 0.986
\end{tabular}




\section{References}

[1] Ajersch MK, Milner B. Handwriting posture as related to cerebral speech lateralization, sex, and writing hand. Human Neurobiology 1983;2:143-5.

[2] Annett M. A coordination of hand skill and preference replicated. British Journal of Psychology 1976;67:587-92.

[3] Ashe J, Georgopoulos AP. Movement parameters and neural activity in motor cortex and area 5. Cerebral Cortex 1994;4:590-600.

[4] Badian NA, Wolff PH. Manual asymmetries of motor sequencing in boys with reading disability. Cortex 1977;13:343-9.

[5] Bartlett NR, Bartlett SC. Synchronization of a motor response with an anticipated sensory event. Psychological Review 1959;66:203-18.

[6] Boecker H, Kleinschmidt A, Requardt M, Hanicke W, et al. Functional cooperativity of human cortical motor areas during self-paced simple finger movements: a high-resolution MRI study. Brain 1994;117:1231-9.

[7] Brinkman C. Supplementary motor area of the monkey's cerebral cortex: short- and long-term deficits after unilateral ablation and the effects of subsequent callosal section. Journal of Neuroscience 1984;4:918-29.

[8] Chan JL, Ross ED. Left-handed mirror writing following right anterior cerebral artery infarction: evidence for nonmirror transformation of motor programs by right supplementary motor area. Neurology 1988;38:59-63.

[9] Deecke L, Kornhuber HH, Lang W, Lang M, Schreiber H. Timing function of the frontal cortex in sequential motor and learning tasks. Human Biology 1985;4:143-54.

[10] Deecke L, Engel M, Lang W, Kornhuber HH. Bereitschaftspotential preceding speech after holding breath. Experimental Brain Research 1986;65:219-23.

[11] Fraisse P. Rhythm and tempo. In: Deutsch D, editor. The psychology of music. New York: Academic Press, 1982. p. 149-80.

[12] Friston KJ, Jezzard P, Turner R. Movement-related effects in FMR1 time-series. Human Brain Mapping 1994;1:346-55.

[13] Friston KJ, Worsley KJ, Frackowiak RSJ, Mazziofta JC, Evans AC. Assessing the significance of focal activations using their spatial extent. Human Brain Mapping 1994;1:210-20.

[14] Friston KJ, Williams S, Howard R, Frackowiak RS, Turner R. Movement-related effects in FMRI time series. Magnetic Resonance Medicine 1996;35:346-55.

[15] Friston KJ, Holmes AP, Poline JB, Grasby PJ, Williman SCR, Frackowiack RSJ, Turner R. Analysis of fMR time-series revisited. Neuroimage 1998;2:45-53.

[16] Friston KJH, Ashburner J, Poline JB, Frith CD, Heather JD, Frackowiack RSJ Spatial registration and normalization of images. Human Brain Mapping 1995;2:165-189.

[17] Goldberg G. Supplementary motor area structure and function: review and hypotheses. Behavioral-and-Brain-Sciences 1985;8:567-616.

[18] Halsband U, Matsuzaka Y, Tanji J. Neuronal activity in the primate supplementary, pre-supplementary and premotor cortex during externally and internally instructed sequential movements. Neuroscience Research 1994;20:149-55.

[19] Hikosaka O, Sakai R, Miyuauchi S, Takino R, et al. Activation of human presupplementary motor area in learning of sequential procedures: a functional MRI study. Journal of Neurophysiology 1996;76(1):617-21.

[20] Ikeda A, Luders HO, Shibasaki H, Collura TF, Gulyas B. Movement-related potentials associated with bilateral simultaneous and unilateral movements recorded from human supplementary motor area. Electroencephalography and Clinical Neurophysiology 1995;95:323-34.

[21] Jahanshahi M, Jenkins IH, Brown RG, Marsden CD, et al. Self-initiated versus externally triggered movements: I. an inves- tigation using measurement of regional cerebral blood flow with PET and movement-related potentials in normal Parkinson's disease subjects. Brain 1998;118:913-33.

[22] Jäncke L, Specht K, Mirzazade S, Loose R, Himmelbach M, Lutz K, Shah NJ. A parametric analysis of the 'rate effect' in the sensorimotor cortex: a functional magnetic imaging analysis. Neuroscience Letters 1998;252:37-42.

[23] Jäncke L, Peters M, Schlaug G, Posse S, Steinmetz H, MüllerGartner HW. Differential magnetic resonance signal change in human sensorimotor cortex to finger movements of different rate of the dominant and subdominant hand. Cognitive Brain Research 1998;6:279-84.

[24] Kornhuber HH. Attention, readiness for action and the stages of voluntary decision-some electrophysiological correlates in man. In: Creutzfeldt O, Schmidt RF, Willis WD, editors. Sensory-integration in the nervous system. Berlin: SpringerVerlag, 1984. p. 420-9.

[25] Kornhuber HH, Deecke L. The starting function of the SMA. The Behavioral and Brain Sciences 1985;8:591-2.

[26] Larsson J, Gulyas B, Sakai R. Cortical representation of self paced finger movement. Neuroreport 1996;7:463-8.

[27] Liepman H Die linke Hemisphäre und das Handeln, Münchener Medizinische Wochenschrift 1905 52, 2322-2326, 2375-2378.

[28] MacNeilage PF, Studdert-Kennedy MG, Lindblom B. Primate handedness reconsidered. Behavioral and Brain Sciences 1987;10:247-303.

[29] McNabb AW, Carroll WM, Mastaglia FL. 'Alien hand' and loss of bimanual coordination after dominant anterior cerebral artery territory infarction. Journal of Neurology, Neurosurgery and Psychiatry 1988;51:218-22.

[30] Michon J Timing in temporal tracking 1967 Soesterberg, Netherlands, Institute for Perception RAO-TO.

[31] Mesick H, Inane M, Tanji J. Neuronal activity in the primate premotor, supplementary, and precentral motor cortex during visually guided and internally determined sequential movements. Journal of Neurophysiology 1991;66:705-18.

[32] Orgogozo JM, Larsen B. Activation of the supplementary motor area during voluntary movement in man suggests it works as a supramotor area. Science 1979;206:847-50.

[33] Passingham RE. Prefrontal cortex and the sequencing of movement in monkeys (Macaca mulatta). Neuropsychologia 1985;23:453-62.

[34] Peters M. Performance of a rubato-like task - when two things cannot be done at the same time. Music Perception 1985;2:47182.

[35] Peters M. Constraints in the coordination of bimanual movements and their expression in skilled and unskilled subjects. Quarterly Journal of Experimental Psychology 1985;37A:17196.

[36] Peters M. A nontrivial motor performance difference between right-handers and left-handers: Attention as intervening variable in the expression of handedness. Canadian Journal of Psychology 1987;41:91-9.

[37] Peters M, Schwartz S. Coordination of the two hands and effects of attentional manipulation in the production of a bimanual 2:3 polyrhythm. Australian Journal of Psychology 1988;41:215-24.

[38] Peters M. The relationship between variability of intertap intervals and interval duration. Psychological Research 1989;51:3842.

[39] Picard N, Takino R. Motor areas of the medial wall: a review of their location and functional activation. Cerebral Cortex 1996;6:342-53.

[40] Poline JB, Worsley KJ, Holmes AP, Frackowiack RS, Friston KJ. Estimating smoothness in statistical parametric maps: varia- 
bility of $\mathrm{p}$ values. Journal of Computer Assisted Tomography 1995;19:788-96.

[41] Rao SM, Binder JR, Bandettini PA, Hammeke TA, et al. Functional magnetic resonance imaging of complex human movements. Neurology 1993;43:2311-8.

[42] Rao SM, Bandettini PA, Binder JR, Bobholz JA, Hammeke TA, Stein EA, Hyde JS. Relationship between finger movement rate and functional magnetic resonance signal change in human primary motor cortex. Cerebral Blood Flow and Metabolism 1996;16:1250-4.

[43] Roland PE, Zilles K. Functions and structures of the motor cortices in humans. Current Opinions in Neurobiology 1996;6:773-81.

[44] Sadato N, Ibanez V, Deiber MP, Campbell G, Leonardo M, Halleft M. Frequency-dependent changes of regional cerebral blood flow during finger movements. Journal of Cerebral Blood Flow and Metabolism 1996;16:23-33.

[45] Schlaug G, Sanes JN, Thangaraj V, Darby DG, Jäncke L, Edelman RR, Warach S. Cerebral activation covaries with movement rate. Neuroreport 1996;7:879-83.

[46] Stevens LT. On the time sense. Mind 1886;11:393-404.

[47] Talairach J, Tournoux P. Co-planar stereotaxic atlas of the human brain. In: 3-Dimensional proportional system: an approach to cerebral imaging. New York: Thieme Medical Publishers, 1988.

[48] Tanji J, Shima K. Role for supplementary motor area cells in planning several movements ahead. Nature 1994;371:413-6.

[49] Tanji J. The supplementary motor area in the cerebral cortex. Neuroscience Research 1994;19:251-68.
[50] Vanmeter JW, Maisog JM, Zeffiro TA, Halleft M, Herscovitch P, Rapoport SI. Parametric analysis of functional neuroimages: application to a variable-rate motor task. Neuroimage 1995;2:273-83

[51] Vaughn CLD, Webster WG. Bimanual handedness in adults who stutter. Perceptual and Motor Skills 1989;68:375-82.

[52] Wagner C. The influence of tempo of playing on the rhythmic structure studied at pianist's playing scales. In: Vredenbregt $\mathbf{J}$, Wartenweiler J, editors. Medicine and sport, V 6, Biomechanics II. Baltimore: University Park Press, 1971. p. 129-32.

[53] Webster WG. Evidence in bimanual finger-tapping of an attentional component to stuttering. Behavioural Brain Research 1990;37:93-100.

[54] Wiesendanger M, Wicki U, Rouiller E. Are there unifying structures in the brain responsible for interlimb coordination? In: Swinnen S, Heuer $\mathrm{H}$, Massion J, Caesar P, editors. Interlimb coordination. New York: Academic Press, 1994. p. 180-207.

[55] Wolff PH, Cohen C. Dual task performance during bimanual coordination. Cortex 1980;16:119-33.

[56] Woodrow H. The effects of rate sequence upon the accuracy of synchronization. Journal of Experimental Psychology 1932;15:357-79.

[57] Worsley KJ, Friston KJ. Analysis of FMR1 time-series revisited again. Neuroimage 1995;3:173-81.

[58] Yousry TA, Schmid UD, Alkadhi H, Schmidt D, Peraud A, Buettner A, Winkler P. Localization of the motor hand area to a knob on the precentral gyrus. Brain 1997;120:141-57. 